\title{
Olelo Wehewehe no na Mokuna
}

He mau manao wehewehe keia i ka mea heluhelu no na mokuna elima i kakau ia. Ua koho ia he mau olelo noeau kaulana no Niihau i poo manao no kela me keia mokuna no ka wehewehe ana i ka manao nui o kela me keia moolelo. Ua hoopili ia ka manao o ka olelo noeau i ke ano o ka moolelo.

\section{Aia i Niihau Kuu Pawehe, He Moena E Pahee Ai Ko Ili}

I loko o ka moolelo mua, ua hoike ia ko'u pili i ka aina o Niihau, kahi i hanai ia ai mai ko'u wa kamalii mai a hiki i ko'u lilo ana i kanaka makua. Ma laila no au i noho ai me ko'u ohana a ao pu hoi i ka ike o kuu mau kupuna. O kekahi mea hoi nana i hoopili loa i ke keiki i ka aina, kona honua. Ma Niihau, ma hope o ka hanau ana $\mathrm{i}$ ka pepe, lawe ia kona honua a kanu ia ma waenakonu o ka ipuka pa o ka pa hale, kahi i komo ai a i puka ai ka ohana i waho o ka pa hale. O ke kumu no ko lakou kanu ana i ka honua ma ka aina, i mea e lilo ai ia keiki i mahi ai a me ka malama ana i ka aina. He mea hoi ia e hoopili ai i ke keiki i kona aina hanau. A o ka piko o ka pepe, ma ka haule ana mai ka piko mai o ke keiki, ua malama ia i loko o kekahi omole a hiki i ka hele ana o ka ohana i Kauai ma ka uila o Lopikana ma; a laila, ua kiola ia ka piko i loko o ke kai hohonu o Kaulakahi, o ia hoi ke kai ma waena o Niihau a me Kauai. Pela no ka hana a ka poe Niihau me ka manao e lilo ana ia keiki i lawaia. No ia mau kumu, ua hana ia keia mau mea no ka pono o ke keiki i loko o kona ola honua ana ma Niihau.

\section{Ka Wai Huna O Ka Paoo}

I loko o ka moolelo elua, wehewehe ana ia moolelo no ka imi ana i ka ike o na kupuna. O ka mea nui i loko o keia moolelo, o ia hoi ka mahele no ka ninaninau, ke kuleana, a me ka hana haipule. O ka papahana ninaninau, ua malama ia no me ka poe Niihau, no ka mea, na lakou mai na moolelo hai waha e pono ai keia kakau pepa ana. A ma ka olelo kanaka no i malama ia ai 
ka ninaninau ana. Ma mua nae o ka hiki ana mai o ia wa, ua upu maila kekahi ninau, no wai la ia kuleana e kakau ai i ia mau ike o Niihau? I ko'u manao, ua upu mai keia wahi olelo, ina he moolelo no Niihau, na ka Niihau no e hoike pololei aku i ko lakou moolelo ponoi. Aole ia kuleana i ka poe o waho. O ka mahele hope o keia moolelo, o ia hoi ka hana haipule. Ma Niihau, he mea nui ka hana haipule, a ma na mea a pau a ka poe Niihau e hana ai, ua hoomaka ia a hookuu ia no me ka pule.

\section{Niihau I Ke Kiku}

I loko o ka moolelo ekolu, pili no i ka olelo kanaka, o ia hoi ka olelo i hanai ia I ka poe Niihau mai ka wa kahiko a hiki i keia la. Hoike ia ke ano o ka olelo Niihau me ka t, ka puana ana, ka puana pokole ana, na hua olelo Niihau, ke kakau ana, na olelo nane, na olelo noeau, ka olelo kake, a me na moolelo no Niihau. I ke kuai ana ia o Niihau e ka ohana Sinclair i ka makahiki 1864, ua ao mai na haku aina i ka olelo kanaka i wahi no lakou e kamailio ai me ka poe Niihau. I ka wa ma mua, ua kipa na malihini olelo kanaka i Niihau, a ua kamailio lakou me ka poe Niihau ma ka olelo kanaka.

\section{Ke Ko Eli Lima O Halalii}

I loko o ka moolelo eha, ua koho ia o "Ke ko eli lima o Halalii" mamuli o ke kaukai ana o ka poe Niihau i ka laau lapaau. Mamuli o ka loaa ole ana o ke kauka lapaau ma Niihau, ua kaukai ka poe Niihau i ka laau lapaau no ka lapaau ana i ko lakou mai. No ia kumu, he nui na laau lapaau a ka poe Niihau i hoohana ai no kela ano mai keia ano mai. O na laau i hoohana ia, o ia hoi na laau i loaa ma Niihau. Na ko'u kupunakane, o Ernest Enoka Kaohelaulii, i hoomakaukau i ka laau lapaau na ka ohana. O kekahi kuleana ona, ka hoomakaukau ana i ka laau lapaau i kapa ia he apu hala no na wahine hanau o Niihau. I ko'u wa kamalii, he mea maamau ke kau mau o ka maka o ka poe Niihau i ko'u kupunakane no ka hoomakaukau ana i ka laau lapaau e like me ka apu hala, ka popolo a me ke kukae puaa, a me ka pohuli maia. Mamuli hoi o ka ike o ko'u kupunakane i loko o ia mahele, ua ili mai kona ike ma luna o kana mau pulapula.

\section{Na Ulu Hua I Ka Hapapa}

I loko o ka moolelo elima, pili no i ka hanau keiki ana ma Niihau. Ua koho ia o $\mathrm{Na}$ Ulu Hua i ka Hapapa i poomanao no keia mokuna mamuli o ka manao o "hua." Ua koho ia keia poomanao i kumu e wehewehe aku ai i ka hanau keiki ana 
ma Niihau. O kekahi kumu i manao ia ai i mea e hoike ai i ka lehulehu na na kupuna no o Niihau i malama i ka hanau keiki ana ma Niihau. O Niihau, aohe haukapila a aohe kauka lapaau e like pu me ko waho nei. No laila, na kela me keia kupuna i loko o kela me keia ohana, na lakou no i malama i ka mahele hoohanau keiki. Wahi a Mileka Kanahele, i kona wa opio, na kona makuakane o Kumuwaina Kanahele i hoohanau i na wahine hanau keiki o Niihau. Oiai, o ia ka hanau mua i loko o kona ohana, nana no i kokua aku i kona makuakane ma ka hoomakaukau ana i na mea e pono ai ka hanau keiki ana. O kana hana, o ia hoi ka hoowela ana i ka wai wela i loko o ke kapu a i ole ke kini huina ha. Ua hoohana ia ka wai wela ina hanau ia mai ke keiki a hauliuli kona ili mamuli hoi o ke anuanu. O ka wai wela ka mea nana i hoopumehana hou i ke kino o ke keiki. O ka mea e ike ai i ka mehana hou ana mai o ke kino o ke keiki, ka hele ana a haulaula ke nana aku. Oiai, okoa ka hanau ana a ka poe Niihau, ua manao au he mea nui ka hoike ana i keia ike hanau keiki o ka poe Niihau i loko o keia moolelo i hiki i ko waho nei ke hoohana aku i kela mau ike hanau keiki ina pono. 
